\title{
Flute mode fluctuations in the divertor mirror cell
}

\author{
I. Katanuma, ${ }^{\text {a) }}$ K. Yagi, Y. Nakashima, M. Ichimura, and T. Imai \\ Plasma Research Center, University of Tsukuba, Tsukuba, Ibaraki 305-8577, Japan
}

(Received 31 August 2009; accepted 18 January 2010; published online 8 March 2010)

\begin{abstract}
The computer code by reduced magnetohydrodynamic equations were made which can simulate the flute interchange modes (similar to the Rayleigh-Taylor instability) and the instability associated with the presence of nonuniform plasma flows (similar to the Kelvin-Helmholtz instability). This code is applied to a model divertor and the GAMMA10 [M. Inutake et al., Phys. Rev. Lett. 55, 939 (1985)] with divertor in order to investigate the flute modes in these divertor cells. The linear growth rate of the flute instability determined by the nonlocal linear analysis agrees with that in the linear phase of the simulations. There is a stable nonlinear steady state in both divertor cells, but the nonlinear steady state is different between the model divertor and the GAMMA10 with divertor.
\end{abstract}

(C) 2010 American Institute of Physics. [doi:10.1063/1.3310838]

\section{INTRODUCTION}

The nonaxisymmetric mirror cell such as a minimum-B mirror breaks the omnigenity property ${ }^{1}$ of a tandem mirror, ${ }^{2,3}$ where the omnigenity means that all drift surfaces of ions with different $\varepsilon$ (kinetic energy) and $\mu$ (magnetic moment) lies on the same flux surface. The magnetic field in which the ions with different $\varepsilon$ and $\mu$ have the different drift surfaces generally causes the neoclassical radial transport. ${ }^{4-8}$

The nonaxisymmetric (but effectively axisymmetrized) GAMMA10 tandem mirror ${ }^{9}$ is, therefore, expected to be axisymmetrized in keeping the magnetohydrodynamic (MHD) stable character. The path toward the MHD stable axisymmetric mirror, especially stable for a flute interchange mode, is briefly mentioned in the following. When two neighboring magnetic flux tubes with plasma are exchanged in an open magnetic system, the plasma internal energy $Q_{p}$ changes by ${ }^{10}$

$$
\delta Q_{p}=\delta p \delta V+\gamma p \frac{(\delta V)^{2}}{V}>0 .
$$

Here $\delta p$ represents the magnitude of outward change of the plasma pressure and $\gamma=5 / 3$ is the ratio of specific heats for an ideal gas with three degrees of freedom. The outward change of plasma volume $\delta V$ is written as $\delta V=\Phi_{m} \delta U$ in terms of a unit magnetic flux $\Phi_{m}$ and the magnetic specific volume defined by

$$
U \equiv \int \frac{\mathrm{d} \zeta}{B}
$$

where $B$ is magnetic field and the integration is carried out along a magnetic field line. The condition $\delta Q_{p}>0$ is required for the stability of flute modes as already written in Eq. (1). In the traditional tandem mirror, in which a long thin approximation is assumed in analysis, the second term of Eq. (1) can be neglected compared with the first term, so that $\delta Q_{p} / \Phi_{m}=\delta p \delta U>0$.

The stability condition is $\delta U<0$ because $\delta p<0$ in

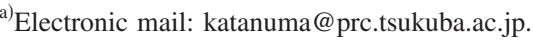

the typical experimental device. Noting that the vacuum magnetic field satisfies $\nabla_{\perp} B=\boldsymbol{\kappa} B$, it is shown that $\partial U / \partial \psi$ $=-2 \int\left(\kappa_{\psi} / B\right) \mathrm{d} \zeta$ in the flux coordinate $(\psi, \theta, \zeta)$, where $\boldsymbol{B}=\nabla \psi \times \nabla \theta$, the magnetic field line curvature $\boldsymbol{\kappa} \equiv \hat{\boldsymbol{e}}_{\|} \cdot \nabla \hat{\boldsymbol{e}}_{\|}$ $=\kappa_{\psi} \nabla \psi+\kappa_{\theta} \nabla \theta$ and $\hat{e}_{\|} \equiv \boldsymbol{B} / B$. Therefore the stability criterion of flute modes is obtained in a low $\beta$ limit, where $\beta$ is the plasma pressure divided by the magnetic pressure, as ${ }^{11}$

$$
\int \frac{\kappa_{\psi} \mathrm{d} \zeta}{B}>0
$$

Equation (3) indicates that the minimum-B mirror cell is required to the stability. Especially in the system with anisotropic pressure $p_{\perp} \neq p_{\|}$, the stability criterion (3) is modified to be $\mathrm{e}^{12-14}$

$$
\int \frac{\left(\hat{p}_{\perp}+\hat{p}_{\|}\right) \kappa_{\psi} \mathrm{d} \zeta}{B}>0
$$

Here the plasma pressures $p_{\perp, \mid l}(\psi, B)$ are represented by separation of variables $p_{\perp, \|}(\psi, B)=\hat{p}_{\perp, \|}(B) \nu(\psi)$. The variables $\hat{p}_{\perp, \|}(B)$ mean the pressure profiles along a magnetic field line (i.e., with $\psi$ fixed). The variable $\nu(\psi)$ is the pressure radial profile at the central cell midplane of a tandem mirror where $B$ is almost constant radially. Equation (4) is also derived from $\partial U / \partial \psi<0$ by defining

$$
U \equiv \int \frac{\left(\hat{p}_{\perp}+\hat{p}_{\|}\right) \mathrm{d} \zeta}{B},
$$

in place of Eq. (2). Equation (4) introduced the idea of average minimum-B stabilization and it was used to design the nonaxisymmetric GAMMA10 tandem mirror for stabilizing the flute modes.

When an equilibrium plasma flow exists along magnetic lines, the flow energy $\rho_{m} v^{2}$, where $\rho_{m}$ is mass density and $v$ is the flow velocity, plays a role of parallel pressure, that is, $\left(\hat{p}_{\perp}+\hat{p}_{\|}\right)$is replaced by $\left(\hat{p}_{\perp}+\hat{p}_{\|}+\rho_{m} v^{2}\right)$ in Eq. (4). In a fully axisymmetric mirror, the normal curvature is written as $\kappa_{\psi}=\left(\mathrm{d}^{2} r / \mathrm{d} z^{2}\right) /(r B)$, where $r$ and $z$ are the radial and axial positions of a magnetic field line. Applying the stability criterion to the outermost magnetic flux tube $r=a$ with the con- 

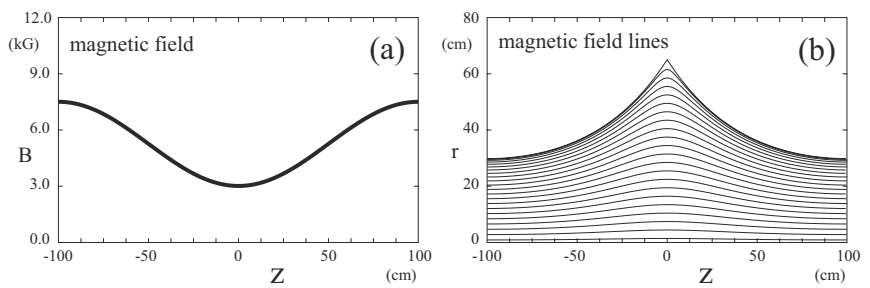

FIG. 1. Model magnetic divertor used in the simulation. (a) is the axial magnitude of magnetic field and (b) is the magnetic field lines.

servation of magnetic flux inside the outermost magnetic flux tube, the stability criterion for GDT ${ }^{15}$ is obtained as

$$
\begin{aligned}
& \int \frac{\mathrm{d}^{2} a}{\mathrm{~d} z^{2}} \frac{\left(\hat{p}_{\perp}+\hat{p}_{\|}+\rho_{m} v^{2}\right) \mathrm{d} \zeta}{a B^{2}} \\
& \quad=\frac{1}{\left(B a^{2}\right)^{2}} \int a^{3} \frac{\mathrm{d}^{2} a}{\mathrm{~d} z^{2}}\left(\hat{p}_{\perp}+\hat{p}_{\|}+\rho_{m} v^{2}\right) \mathrm{d} \zeta>0 .
\end{aligned}
$$

The idea of the gas dynamic trap (GDT) makes use of the good curvature region just outside the outermost mirror throat of the mirror machine where the large plasma loss flux exists, and it can contribute to the MHD stability in a high density operation. It has been reported in the GDT experiment $^{16,17}$ that the plasma was sustained stably.

The similar approach to the axisymmetric mirror is proposed by Post, ${ }^{18}$ where the peaked plasma density in the good curvature region outside the outermost mirror throat is produced by the ion beam injection from the end-wall direction, which stabilizes the confined plasma by its presence.

There is another approach to the axisymmetric mirror which the GAMMA10 tandem mirror have adopted. This is to make a divertor magnetic field inside the GAMMA10 where the case of central cell divertor design was carried out by taking into account the plasma equilibrium. ${ }^{19}$ Historically the equilibrium calculation has been carried out in various divertor mirror cells for isotropic pressure plasma, ${ }^{20}$ for anisotropic pressure plasma ${ }^{19,21}$ and for kinetic KruskalOberman stability theory with anisotropic pressure. ${ }^{22}$ The theoretical study of flute mode stability by a magnetic divertor was done first by Lane et al. ${ }^{23}$ The subsequently the theory was extended by Pastukhov and Sokolov ${ }^{24}$ by taking into account the nonparaxial magnetic filed line curvature and then was extended further more by Sasagawa et al. ${ }^{25}$ by including the anisotropic ion temperature effects. The general concept of divertor stabilization in mirror-based plasma confinement systems were also discussed by Pastukhov. ${ }^{26}$ The experimental studies were also carried out by the tandem mirrors. $^{27-29}$

In the following the MHD stability mechanism by a magnetic divertor is mentioned briefly. The magnetic divertor has a magnetic null at the separatrix in Fig. 1(b). The electrons around the magnetic null are unmagnetized so that they can move freely in the azimuthal direction which have a short circuit effect to the perturbed electrostatic potential around the separatrix. Another stabilizing effect is a plasma compression. The specific volume $U$ is infinitely large at the separatrix so that the second term cannot be neglected in Eq. (1) and then the stability criterion becomes

$$
\delta Q_{p} / \Phi_{m}=\delta U \delta\left(p U^{\gamma}\right) / U^{\gamma}>0 .
$$

Because $\delta U>0$ in the neighborhood of the separatrix, the stability criterion is $\delta\left(p U^{\gamma}\right)>0$. Especially the condition $\partial\left(p U^{\gamma}\right) / \partial \psi=0$ gives the marginal stability pressure radial profile. Noting that $U \rightarrow \infty$ at the separatrix, the condition $p U^{\gamma}=$ const. gives $p \rightarrow 0$ at the separatrix which meets the condition that the pressure is 0 at the limiter in an experimental device.

It is important to understand the flute mode fluctuations in the divertor mirror cell even if the flute modes are stabilized by the pressure radial profile of $p U^{\gamma}=$ const. because there is a magnetic null region where a large radial transport exist due to a large ion Larmor radius there. That is, a large diffusion forms locally stable pressure profile in the neighborhood of magnetic null and forms unstable pressure profile outside this area where the relation $p U^{\gamma}=$ const. is broken, so the system can become unstable to the flute mode. The purpose of this article, therefore, is to investigate the flute mode fluctuations in the nonlinear state in a magnetic divertor numerically.

\section{BASIC EQUATIONS}

In this section we describe the basic equations by which the nonlinear MHD convection, the flute instability, and flute mode fluctuations are investigated, where the detailed derivation of the following basic equations, which are in the framework of MHD, is given in the references. ${ }^{30,31}$ The coordinates adopted here are $(x, \varphi, \zeta)$, where $x \equiv \sqrt{\psi / \psi_{b}}$ with $\psi(r, z) \equiv \int_{0}^{r} B_{z}\left(r^{\prime}, z\right) r^{\prime} \mathrm{d} r^{\prime}, \varphi$ is the azimuthal coordinate, and $\zeta$ is a coordinate along a magnetic field line. The quantity $2 \pi \psi_{b}$ is the magnetic flux surrounded by the outermost magnetic flux tube (separatrix), on which magnetic field lines pass through a magnetic null point.

The specific dynamic vorticity $\hat{w}$, mass density $\hat{\rho}$, and effective temperature $\hat{T}$, which are the quantities integrated over a unit magnetic flux tube, are defined as ${ }^{30}$

$$
\begin{aligned}
& \hat{w} \equiv \frac{\partial}{\partial \psi}\left(\hat{\rho}\left\langle r^{2}\right\rangle \frac{\partial \Phi}{\partial \psi}\right)+\frac{\partial}{\partial \varphi}\left(\hat{\rho}\left\langle\frac{1}{r^{2} B^{2}}+\lambda^{2} B^{2}\right\rangle \frac{\partial \Phi}{\partial \varphi}\right), \\
& \hat{\rho} \equiv \int \frac{\rho \mathrm{d} \zeta}{B}=\rho U, \\
& \hat{T} \equiv\left(T_{i}+T_{e}\right) U^{\gamma-1} / M_{i}=\left(T_{i}+T_{e}\right) U^{2 / 3} / M_{i},
\end{aligned}
$$

where $\rho, T_{e}$, are $T_{i}$ are the local mass density, electron, and ion temperatures, all of which are assumed to be constant along a magnetic field line, $\Phi$ is the electrostatic potential multiplied by light speed $c$ and $\lambda=U(\partial / \partial \psi)\left(\int_{0}^{\zeta}[1 / U J] \mathrm{d} \zeta\right)$ $+(\nabla \psi \cdot \nabla \zeta) /\left(r^{2} B^{2} J\right), J=\nabla \psi \times \nabla \varphi \cdot \nabla \zeta$. The adiabatic velocity $\boldsymbol{v}_{\alpha}$ of plasma has the form of

$$
\boldsymbol{v}_{\alpha}=\frac{-\nabla \Phi \times \boldsymbol{B}}{B^{2}}+\boldsymbol{B} \lambda \frac{\partial \Phi}{\partial \varphi},
$$

where the first and second terms in the right-hand side are the velocities across and along a magnetic field line, and the 
velocity satisfies $\nabla \cdot\left(\boldsymbol{v}_{\alpha} / U\right)=0$, which is the condition of incompressible flow of mass density $\hat{\rho}$.

The basic equations described in this section can be applied under the assumption that the plasma pressure is below the instability threshold for Alfvén modes $\left(\beta<\beta_{\mathrm{cr}} \sim 1\right)$. And the high-frequency stable collective degrees of freedom corresponding to magnetosonic, Alfvén, and longitudinal acoustic modes are excluded from the basic equations. ${ }^{30}$ The effects of magnetic field line curvature on the flute interchange modes are included through the specific volume $U$, so that the quantities in Eq. (8) are a function of $\psi$ and $\varphi$.

The normalized quantities are used in the following which are used in the simulation code. The normalized quantities $w, D$, and $T$, which correspond to the specific dynamic vorticity $\hat{w}$, mass density $\hat{\rho}$, effective temperature $\hat{T}$, respectively, are described by a sum of zeroth and perturbed quantities as

$$
\begin{aligned}
& w(x, \varphi)=w_{0}(x)+w_{f}(x, \varphi), \\
& D(x, \varphi)=D_{E}(x)+\epsilon^{2} D_{f}(x, \varphi), \\
& T(x, \varphi)=T_{E}(x)+\epsilon^{2} T_{f}(x, \varphi) .
\end{aligned}
$$

Here $\epsilon^{2}$ is a small expansion parameter defined by $\epsilon^{2} \equiv\left(\chi_{M} / b c_{s M}\right)\left[2 T_{i} /\left(T_{i}+T_{e}\right)\right]_{M}^{1 / 2}, \quad$ where $\quad c_{s M}=\sqrt{\gamma p_{M} / \rho_{M}}$, $b=\sqrt{\psi_{b} / B_{M}}$, and $\chi_{M}=\left[T_{i} /\left(M_{i} \omega_{c i} \tau_{i}\right)\right]_{M}$ which is related to the classical thermal diffusitivity. The quantities $p, M_{i}, \omega_{c i}$, and $\tau_{i}$ are pressure, ion mass, ion cyclotron frequency, and ion coulomb collision time, respectively. The subscript ${ }_{M}$ means the quantity at the midplane on axis of the divertor mirror cell.

The zeroth quantities $w_{0}(x), D_{E}(x)$, and $T_{E}(x)$ are averaged over $\varphi$

$$
\begin{aligned}
\frac{\partial w_{0}}{\partial \tau}= & \frac{1}{2 x} \frac{\partial}{\partial x}\left(\overline{w_{f} \frac{\partial \phi}{\partial \varphi}}+\frac{\epsilon^{2}}{2} \overline{D_{f} \frac{\partial V^{2}}{\partial \varphi}}\right) \\
& +\frac{\epsilon^{2} C_{2}}{4 x} \frac{\partial}{\partial x}\left[\frac{f_{1} D_{E}}{x} \frac{\partial}{\partial x}\left(\frac{f_{2}}{u^{2 / 3} f_{1}^{2} \sqrt{T_{E}}} w_{0}\right)\right]+u Q_{w 0}, \\
\frac{\partial D_{E}}{\partial \tau}= & \frac{\epsilon^{2}}{2 x} \frac{\partial}{\partial x}\left(\overline{D_{f} \frac{\partial \phi}{\partial \varphi}}\right)+\frac{\epsilon^{2} C_{1}}{4 x} \frac{\partial}{\partial x}\left[\frac{u f_{1} D_{E}}{x T_{E}^{3 / 2}} \frac{\partial}{\partial x}\left(\frac{D_{E} T_{E}}{u^{5 / 3}}\right)\right] \\
& +\epsilon^{2} u Q_{D 0}, \\
\frac{\partial T_{E}}{\partial \tau}= & \epsilon^{2} \frac{1}{2 x} \frac{\partial}{\partial x}\left(\overline{T_{f}} \frac{\partial \phi}{\partial \varphi}\right) \\
& +\frac{\epsilon^{2}}{12} \frac{u^{2 / 3}}{D_{E}} \frac{1}{x} \frac{\partial}{\partial x}\left[\frac{f_{1} D_{E}^{2}}{x u^{2 / 3} \sqrt{T_{E}}} \frac{\partial}{\partial x}\left(\frac{T_{E}}{u^{2 / 3}}\right)\right] \\
& +\frac{C_{1} \epsilon^{2}}{6 x \sqrt{T_{E}}} \frac{\partial}{\partial x}\left[\frac{u f_{1}}{x} \frac{\partial}{\partial x}\left(\frac{D_{E} T_{E}}{u^{5 / 3}}\right)\right]+\frac{10}{9} C_{2} \epsilon^{4} \frac{f_{2}\left(w^{2}\right)_{0}}{f_{1}^{2} D_{E} \sqrt{T_{E}}} \\
& +C_{1} \epsilon^{2} \frac{f_{1}}{T_{E}^{3 / 2}}\left[\frac{1}{2 x} \frac{\partial}{\partial x}\left(\frac{D_{E} T_{E}}{u^{5 / 3}}\right)\right]+\epsilon^{2} \frac{u^{5 / 3}}{D_{E}} Q_{T 0} .
\end{aligned}
$$

time, the constants are $C_{1}=\sqrt{2 M_{e} / M_{i}}$ and $C_{2}=3 \sqrt{2} / 20$ on the assumption of $T_{e}=T_{i}$, where $M_{e}$ is the electron mass, $f_{1}$ $=\left\langle r^{2}\right\rangle / b^{2}$ and $f_{2}=\left\langle r^{4}\right\rangle / b^{4}$. The symbol $\langle\ldots\rangle$ means the average over a magnetic flux tube, that is $\langle A\rangle \equiv(1 / U) \int(A / B) \mathrm{d} \zeta$ where $B$ is magnetic field. The overline $\bar{A}$ means the average of $A$ over $\varphi$, i.e., $\bar{A} \equiv \int_{0}^{2 \pi} A \mathrm{~d} \varphi / 2 \pi$. The first terms in the right-hand side of Eqs. (11)-(13) are the radial transport coming from the unstable fluctuation of the perturbed quantities. The second terms in the right-hand side of Eqs. (11) and (12) and the second and third terms in the right-hand side of Eq. (13) are the classical diffusion process. ${ }^{32}$ The fourth and fifth terms in the right-hand side of Eq. (13) are the viscous heating and Joule heating, respectively, which are negligibly small in this article. The last terms $Q_{w 0}, Q_{D 0}$, and $Q_{T 0}$ in the above equations are the effective source terms of specific dynamic vorticity, density, and energy. The specific volume $u(x)$ is normalized as

$$
u(x) \equiv U(x) / U(0) .
$$

The equations of the perturbed components $w_{f}(x, \varphi)$, $D_{f}(x, \varphi)$, and $T_{f}(x, \varphi)$, which have the dependence on the azimuthal coordinate $\varphi$, are written as, respectively,

$$
\begin{aligned}
\frac{\partial w_{f}}{\partial \tau}+ & \llbracket \phi, w_{f} \rrbracket-\frac{1}{2 x} \frac{\partial \phi}{\partial \varphi} \frac{\partial w_{0}}{\partial x}-\frac{\epsilon^{2}}{2} \llbracket D_{f}, V^{2} \rrbracket-\frac{1}{4 x} \frac{\partial V^{2}}{\partial \varphi} \frac{\partial D_{E}}{\partial x} \\
+ & \frac{3}{10 x} \frac{1}{u^{5 / 3}} \frac{\partial u}{\partial x} \frac{\partial\left(D_{E} T_{f}+T_{E} D_{f}\right)}{\partial \varphi} \\
= & \epsilon^{2} C_{2}\left\{\frac { 1 } { 4 x } \frac { \partial } { \partial x } \left[\frac { D _ { E } f _ { 1 } } { x } \frac { \partial } { \partial x } \left(\frac{f_{2}}{\left.\left.u^{2 / 3} f_{1}^{2} \sqrt{T_{E}} w_{f}\right)\right]}\right.\right.\right. \\
& \left.+\frac{D_{E} f_{2}\left(f_{3}+f_{4}\right)}{u^{2 / 3} f_{1}^{2} \sqrt{T_{E}}} \frac{\partial^{2} w_{f}}{\partial \varphi^{2}}\right\}+u \widetilde{Q}_{w}, \\
\frac{\partial D_{f}}{\partial \tau}+ & \llbracket \phi, D_{f} \rrbracket-\frac{1}{2 \epsilon^{2} x} \frac{\partial \phi}{\partial \varphi} \frac{\partial D_{E}}{\partial x} \\
= & \frac{\epsilon^{2} C_{1}}{4 x} \frac{\partial}{\partial x}\left[\frac{u f_{1}}{x} \frac{D_{E}}{T_{E}^{3 / 2}} \frac{\partial}{\partial x}\left(\frac{D_{f} T_{E}}{u^{5 / 3}}\right)\right] \\
& +\epsilon^{2} C_{1} \frac{f_{3} D_{E}}{u^{2 / 3} \sqrt{T_{E}}} \frac{\partial^{2} D_{f}}{\partial \varphi^{2}}+u \widetilde{Q}_{D},
\end{aligned}
$$

$$
\begin{aligned}
\frac{\partial T_{f}}{\partial \tau}+\llbracket \phi, T_{f} \rrbracket-\frac{1}{2 \epsilon^{2} x} \frac{\partial \phi}{\partial \varphi} \frac{\partial T_{E}}{\partial x} \\
=\epsilon^{2} C_{3} \frac{u^{2 / 3}}{12 x D_{E}} \frac{\partial}{\partial x}\left[\frac{D_{E}^{2} f_{1}}{x u} \frac{\partial}{\partial x}\left(\frac{T_{f}}{u^{1 / 3} \sqrt{T_{E}}}\right)\right] \\
+\epsilon^{2} C_{3} \frac{D_{E} f_{3}}{3 u^{2 / 3} \sqrt{T_{E}}} \frac{\partial^{2} T_{f}}{\partial \varphi^{2}}+\frac{10 \epsilon^{2}}{9} C_{2} \frac{f_{2}\left(w^{2}\right)_{f}}{f_{1}^{2} D_{E} \sqrt{T_{E}}}+\frac{u^{5 / 3}}{D_{E}} \widetilde{Q}_{T} .
\end{aligned}
$$

Here the normalized Poisson bracket is defined by 


$$
\llbracket \Phi, F \rrbracket \equiv \frac{1}{2 x} \frac{\partial \Phi}{\partial x} \frac{\partial F}{\partial \varphi}-\frac{1}{2 x} \frac{\partial \Phi}{\partial \varphi} \frac{\partial F}{\partial x} .
$$

The left-hand side of Eq. (15) contains the terms describing the ideal plasma dynamics, and the right-hand side includes the classical diffusion process and the effective source term of specific dynamic vorticity $\widetilde{Q}_{w}$. The equation of continuity of mass density results in Eq. (16), and the first and second terms in the right-hand side are the classical diffusion, and third term is the effective mass density source $\widetilde{Q}_{D}$. Equation (17) is derived from the transport equation of plasma pressure in the one fluid MHD model in accordance with the classical review by Braginskii. ${ }^{32}$ The first and second terms in the right-hand side in Eq. (17) are the classical diffusion, the third and fourth terms are the heating due to the flow in the viscous fluid which are negligibly small in the article, and $\widetilde{Q}_{T}$ is the effective heat source of joule heating and external heating.

The normalized electrostatic potential $\phi$ is determined by Eq. (8), where the specific dynamic vorticity $w_{0}+w_{f}$ includes the term $\phi$, which gives the following equation:

$$
\begin{gathered}
\frac{1}{x} \frac{\partial}{\partial x}\left(\frac{D_{E} f_{1}}{x} \frac{\partial \phi}{\partial x}\right)+4 \frac{\partial}{\partial \varphi}\left[D_{E}\left(f_{3}+f_{4}\right) \frac{\partial \phi}{\partial \varphi}\right]=4\left(w_{0}+w_{f}\right) \\
-\frac{\epsilon^{2}}{x} \frac{\partial}{\partial x}\left(\frac{D_{f} f_{1}}{x} \frac{\partial \phi}{\partial x}\right)-4 \epsilon^{2} \frac{\partial}{\partial \varphi}\left[D_{f}\left(f_{3}+f_{4}\right) \frac{\partial \phi}{\partial \varphi}\right] .
\end{gathered}
$$

The normalized square of flow velocity $V^{2}$ averaged over a magnetic flux tube is given by

$$
\begin{aligned}
V^{2}= & \frac{\left\langle r^{2}\right\rangle}{b^{2}}\left(\frac{1}{2 x} \frac{\partial \phi}{\partial x}\right)^{2}+\left(\left\langle\frac{1}{r^{2} B^{2}}\right\rangle B_{M}^{2} b^{2}+\left\langle\lambda^{2} B^{2}\right\rangle B_{M}^{2} b^{2}\right) \\
& \times\left(\frac{\partial \phi}{\partial \varphi}\right)^{2}
\end{aligned}
$$

Here $C_{3}=\sqrt{2}$ on the assumption of $T_{e}=T_{i}, f_{3}$ $=\left\langle\left(r^{2} B^{2}\right)^{-1}\right\rangle B_{M} \psi_{b}$, and $f_{4}=\left\langle\lambda^{2} B^{2}\right\rangle B_{M} \psi_{b}$.

\section{LINEAR ANALYSIS}

The basic Eqs. (15)-(17) and (19) with (11)-(13) gives a set of equations for the nonlinear MHD convection. The linear analysis is helpful to understand those equations. The perturbed function $w_{f}$ is expanded in Fourier series as

$$
w_{f}=\sum_{m} w_{f(m)} \exp \{-\mathrm{i} \omega \tau+\mathrm{i} m \varphi\}
$$

Here $\mathrm{i} \equiv \sqrt{-1}, \omega$ is a frequency of linear wave, and $m$ is an azimuthal mode number. The remaining unknown functions $D_{f}, T_{f}$, and $\phi$ are expanded in the same way.

Assuming that the functions $\phi, w_{f}, D_{f}$, and $T_{f}$ are independent of $x$, the local dispersion is obtained from Eqs. (15)-(17) and (19) as

$$
\begin{aligned}
(\omega- & \left.\frac{m}{2 x} \frac{\partial \phi_{0}}{\partial x}\right)^{2} m^{2} D_{E}\left(f_{3}+f_{4}\right)+\left(\omega-\frac{m}{2 x} \frac{\partial \phi_{0}}{\partial x}\right) \\
& \times\left[\frac{m}{8 x^{2}} \frac{\partial D_{E}}{\partial x} \frac{\partial}{\partial x}\left(\frac{f_{1}}{x} \frac{\partial \phi_{0}}{\partial x}\right)-\frac{m}{2 x} \frac{\partial w_{0}}{\partial x}\right] \\
& -\left[\frac{m^{2}}{8 x^{2}} \frac{\partial\left(V^{2}\right)_{0}}{\partial x} \frac{\partial D_{E}}{\partial x}+\frac{3 m^{2}}{20 u^{5 / 3} \epsilon^{2} x^{2}} \frac{\partial u}{\partial x} \frac{\partial\left(D_{E} T_{E}\right)}{\partial x}\right]=0 .
\end{aligned}
$$

Here the classical diffusion terms and source terms in the right-hand sides of Eqs. (15)-(17) and (19) are neglected in Eq. (22).

Equation (22) shows that the system is unstable to the flute interchange modes (similar to the Rayleigh-Taylor instability) in the case of $(\partial u / \partial x)\left(\partial D_{E} T_{E} / \partial x\right)<0$, when $\partial D_{E} / \partial x=\partial w_{0} / \partial x=0$. The term $\left[\partial\left(V^{2}\right)_{0} / \partial x\right]\left(\partial D_{E} / \partial x\right)$ is responsible for a centrifugal drive. The instability associated with the presence of nonuniform plasma flows (similar to the Kelvin-Helmholtz instability) comes from the term proportional to $\partial w_{0} / \partial x$ (similar to the second derivative of shearflow velocity in incompressible fluid). There are no solutions corresponding to the magnetosonic, Alfvén, and longitudinal acoustic modes in Eq. (22) because these high-frequency stable modes are excluded from the basic equations as already mentioned in Sec. II.

Because the geometrical coefficients $f_{3}, f_{4}$, and $\partial u / \partial x$ are generally a function of $x$ in Eq. (22), the local approximation is not suitable. Instead a nonlocal treatment is required, by which the dispersion equation is obtained as

$$
\begin{aligned}
\frac{\partial}{\partial x}( & \left.\frac{D_{E} f_{1}}{x} \frac{\partial \phi_{(m)}}{\partial x}\right)-\frac{\partial}{\partial x} \\
& \times\left[\frac{m f_{1}}{2 x^{2}} \phi_{(m)} \frac{\partial \phi_{0}}{\partial x} \frac{\partial D_{E}}{\partial x} /\left(\omega-\frac{m}{2 x} \frac{\partial \phi_{0}}{\partial x}\right)\right] \\
& -\left[4 x D_{E}\left(f_{3}+f_{4}\right) m^{2}+\frac{4 x w_{f(m)}}{\phi_{(m)}}\right] \phi_{(m)}=0 .
\end{aligned}
$$

Here $\phi_{0}(x)$ is determined by

$$
\frac{\partial}{\partial x}\left(\frac{D_{E} f_{1}}{x} \frac{\partial \phi_{0}}{\partial x}\right)=4 x w_{0}
$$

and the last term in the left-hand side of Eq. (23) is written as

$$
\begin{aligned}
\frac{4 x w_{f(m)}}{\phi_{(m)}}= & -\frac{m^{2}}{2 x} \frac{\partial\left(V^{2}\right)_{(0)}}{\partial x} \frac{\partial D_{E}}{\partial x} /\left\{\left(\omega-\frac{m}{2 x} \frac{\partial \phi_{0}}{\partial x}\right)\right. \\
& \left.\times\left[\omega-\frac{m}{2 x} \frac{\partial \phi_{0}}{\partial x}+\mathrm{i} m^{2} \epsilon^{2} C_{2} \frac{D_{E} f_{2}\left(f_{3}+f_{4}\right)}{u^{2 / 3} f_{1}^{2} \sqrt{T_{E}}}\right]\right\} \\
& -2 m \frac{\partial w_{0}}{\partial x} /\left[\omega-\frac{m}{2 x} \frac{\partial \phi_{0}}{\partial x}+\mathrm{i} m^{2} \epsilon^{2} C_{2}\right. \\
& \left.\times \frac{D_{E} f_{2}\left(f_{3}+f_{4}\right)}{u^{2 / 3} f_{1}^{2} \sqrt{T_{E}}}\right],
\end{aligned}
$$




$$
\begin{aligned}
& -\frac{3 m^{2}}{5 \epsilon^{2} x} \frac{1}{u^{5 / 3}} \frac{\partial u}{\partial x} \frac{\partial\left(D_{E} T_{E}\right)}{\partial x} /\left\{\left(\omega-\frac{m}{2 x} \frac{\partial \phi_{0}}{\partial x}\right)\right. \\
& \left.\times\left[\omega-\frac{m}{2 x} \frac{\partial \phi_{0}}{\partial x}+\mathrm{i} m^{2} \epsilon^{2} C_{2} \frac{D_{E} f_{2}\left(f_{3}+f_{4}\right)}{u^{2 / 3} f_{1}^{2} \sqrt{T_{E}}}\right]\right\},
\end{aligned}
$$

where the second term in the right-hand side of Eq. (15) is taken into account.

Equation (23) is an eigenvalue equation with eigenfunction $\phi_{(m)}$ and eigenvalue $\omega$, which is solved with a boundary condition of $\phi_{(m)}(0)=0$ at $x=0$ and at $x=1$. The solution of Eq. (23) is given in Secs. IV and V.

\section{FLUTE-MODE FLUCTUATIONS IN A MODEL DIVERTOR}

In this section the numerical simulation of flute mode instability in a model divertor given in Eq. (26) is shown

$$
\begin{gathered}
B_{z}=B_{0}\left[1-\frac{1}{I_{0}\left(\pi R_{s} / L\right)} I_{0}(\pi r / L) \cos \left\{\pi \frac{z}{L}\right\}\right], \\
B_{r}=-B_{0} \frac{1}{I_{0}\left(\pi \frac{R_{s}}{L}\right)} I_{1}\left(\pi \frac{r}{L}\right) \sin \left\{\pi \frac{z}{L}\right\} .
\end{gathered}
$$

Here $I_{0}$ and $I_{1}$ are the modified Bessel functions of zeroth order and first order, and the symbol $R_{s}$ is the radial distance from the axis to the magnetic null point. The magnetic field given by Eq. (26) satisfies the periodic boundary condition with period $2 L$ in the $z$ direction. The simulation with the same model configuration in Eq. (26) but different initial conditions from this section has been reported, ${ }^{33}$ where the turbulent structures obtained in the nonlinear phase were briefly mentioned. In the article, $L=100 \mathrm{~cm}$ and $R_{s}=65 \mathrm{~cm}$ are assumed. Figure 1 plots the model divertor magnetic field with above parameters.

The computer simulation, the basic equations of which are given in Sec. II, is carried out to investigate the flute interchange modes in the model divertor magnetic field. The numerical algorithm adopted here is that the CrankNicholson implicit method ${ }^{34}$ is applied to Eqs. (11)-(13), and the Lax-Wendroff scheme ${ }^{34}$ is applied to Eqs. (15)-(17) in the $x$ direction. The fast Fourier transform is used to solve Eqs. (15)-(17) in the $\varphi$ direction. Time step $\Delta t=2 \times 10^{-4}$ and the $(x \times \varphi)=\left(121 \times 2^{6}\right)$ meshes are used. The expansion parameter $\epsilon^{2}=10^{-2}$ is chosen.

The boundary conditions for zeroth quantities are that $\partial D_{E}(0) / \partial x=\partial T_{E}(0) / \partial x=\partial w_{0}(0) / \partial x=0 \quad$ and $\quad \partial D_{E}(1) / \partial x$ $=\partial T_{E}(1) / \partial x=0$. The boundary condition of $w_{0}(x)$ at $x=1$ is chosen that $\int_{0}^{1} w_{0}(x) x \mathrm{~d} x$ is conserved in time. The boundary conditions for perturbed quantities are that $\partial D_{f,(2 m)}(0) / \partial x$ $=\partial T_{f,(2 m)}(0) / \partial x=\partial w_{f,(2 m)}(0) / \partial x=0, \quad D_{f,(2 m+1)}(0)=T_{f,(2 m+1)}(0)$ $=w_{f,(2 m+1)}(0)=0, \quad$ and $\quad \partial D_{f,(m)}(1) / \partial x=\partial T_{f,(m)}(1) / \partial x$ $=\partial w_{f,(m)}(1) / \partial x=0$. These boundary conditions are understood from the following consideration. That is, the perturbed quantity $A_{(2 m)}(x, \theta)$ is proportional to $\exp \{2 m i \theta\}$ and $A_{(2 m+1)}(x, \theta)$ is proportional to $\exp \{(2 m+1) \mathrm{i} \theta\}$. Therefore $A_{(2 m)}(-x, \theta) \equiv A_{(2 m)}(x, \theta+\pi)=A_{(2 m)}(x, \theta)$ and $A_{(2 m+1)}(-x, \theta)$
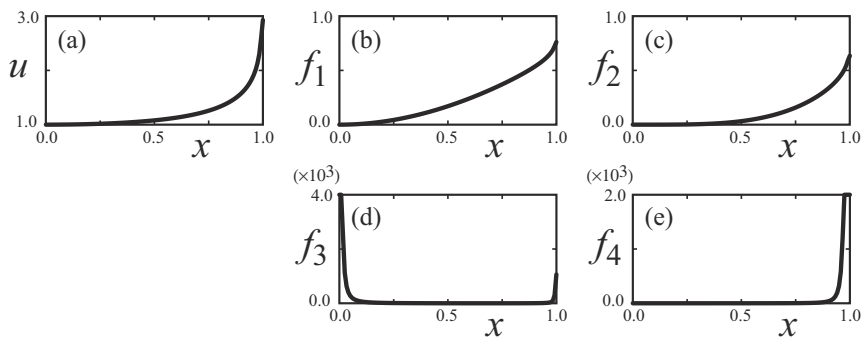

FIG. 2. Coefficients calculated in the model divertor. (a): the normalized specific volume $u(x),(\mathrm{b}): f_{1}(x),(\mathrm{c}): f_{2}(x),(\mathrm{d}): f_{3}(x)$, and (e): $f_{4}(x)$ are plotted, respectively.

$\equiv A_{(2 m+1)}(x, \theta+\pi)=-A_{(2 m+1)}(x, \theta)$, which means that $\partial A_{(2 m)}(0, \theta) / \partial x=0$ and $A_{(2 m+1)}(0, \theta)=0$ are a proper boundary condition at $x=0$. The boundary condition of zeroth quantities $\partial D_{E}(1) / \partial x=\partial T_{E}(1) / \partial x=0$ gives the equilibrium state of $\partial D_{E}(x) / \partial x=\partial T_{E}(x) / \partial x=0$ everywhere if there is no dissipation. Furthermore, for example, one of the boundary conditions $D_{E}(1)$ and $\partial D_{E}(1) / \partial x$ is required in order to calculate $D_{E}(x)$ numerically. We do not want to adopt the fixed $D_{E}(1)$ as a boundary condition because the particle flux through $x=1$ which leads to zero $D_{E}(x)$ everywhere. is necessary for the present calculation. So the remaining boundary condition is $\partial D_{E}(1) / \partial x=C$, where $C$ is constant. Here $D_{E}(x)=\rho_{0}(x) u(x)$, where $\rho_{0}(x)$ is the normalized mass density in Eqs. (8) and (10), gives $\partial D_{E}(1) / \partial x=\rho_{0}(1) \partial u(1) / \partial x$ $+u(1) \partial \rho_{0}(1) / \partial x=C$. In order that $\rho_{0}(1)=\partial \rho_{0}(1) / \partial x=0$ but $u(1)<\infty$ and $\partial u(1) / \partial x<\infty$ just inside the separatrix, $C$ must be 0 , where $x=1$ is not at the separatrix but just inside the separatrix because $u(x)<\infty$ everywhere must be satisfied in the numerical simulation.

Figure 2 plots the normalized specific volume $u(x)$ and other geometrical coefficients $f_{1}(x), f_{2}(x), f_{3}(x)$, and $f_{4}(x)$, where $f_{1}(x), f_{3}(x)$, and $f_{4}(x)$ result from the flux tube averaging procedure in Eq. (8). The feature of the coefficient $f_{3}$ being large around $x=0$ is due to $\nabla_{\varphi}^{2} \propto x^{-2}(\partial / \partial \varphi)^{2}$ in the diffusion term, and that of $f_{3}$ being large around $x \simeq 1$ results from that the classical diffusion is proportional to $B^{-2}$. The coefficient $f_{4}$ is included in Eq. (15) which results from the second term in the right-hand side of Eq. (9), that is the plasma can flow azimuthally along the magnetic null line around the separatrix, as shown in Fig. 2(e). It is assumed that there are not external sources, i.e., $Q_{w 0}(x)=Q_{T 0}(x)$ $=Q_{D 0}(x)=0$ and $\widetilde{Q}_{w}(x)=\widetilde{Q}_{D}(x)=\widetilde{Q}_{T}(x)=0$.

The initial conditions in the simulation are that $D_{E}(x)=1, T_{E}(x)=1$, and $w_{0}(x)=-1$ in $0 \leq x \leq 1$. The system is stable to the flute interchange modes in these initial conditions, as is seen in Eq. (22), where the eigenfrequency $\omega$ is real. The initial perturbation is added to only $T_{f}(x, \varphi)$ such as

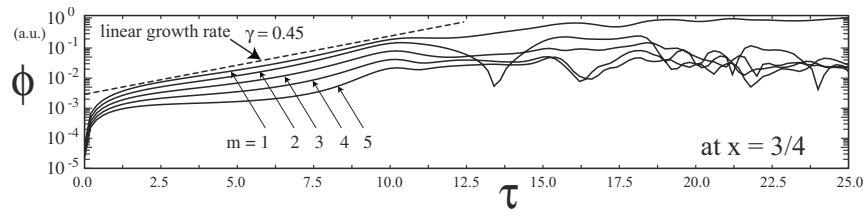

FIG. 3. The time evolution of $m=1-5$ modes of $\phi_{(m)}$ measured at $x=3 / 4$. 

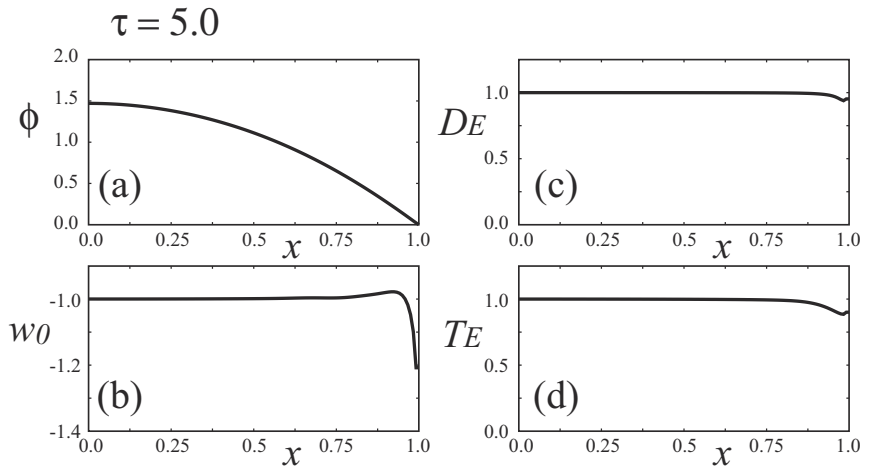

FIG. 4. The radial profiles at $\tau=5$ of (a): potential $\phi$, (b): specific dynamic vorticity $w_{0},(\mathrm{c})$ : mass density $D_{E}$, and (d): effective temperature $T_{E}$.

$$
\begin{aligned}
& T_{f}(x, \varphi)=-\sin \{3 \pi(x-2 / 3)\} \sin \{10 \varphi\}, \\
& 1>x>2 / 3, \quad 0<\varphi<\pi / 10 .
\end{aligned}
$$

Figure 3 plots the time evolution of Fourier amplitude of $\phi_{(m)}$ at $x=3 / 4$. It is seen that the linear grow phase continues during $\tau=1 \sim 10$. In order to calculate the linear growth rate around $\tau \simeq 5$, the radial profiles of $\phi_{0}, w_{0}, D_{E}$, and $T_{E}$ observed in the simulation are plotted in Fig. 4. These radial profiles are approximated by the following functions as

$$
\begin{aligned}
& w_{0}(x)= \begin{cases}-1.0 & : x<0.9 \\
-\exp \left\{+19(x-0.9)^{2}\right\} & : x>0.9\end{cases} \\
& D_{E}(x)= \begin{cases}1.0 & : x<0.9 \\
\exp \left\{-10(x-0.9)^{2}\right\} & : x>0.9\end{cases} \\
& T_{E}(x)= \begin{cases}1.0 & : x<0.85 \\
\exp \left\{-7(x-0.85)^{2}\right\} & : x>0.85\end{cases}
\end{aligned}
$$

The nonlocal eigenvalue in Eqs. (23)-(25) are calculated for $m=1$ mode by using the radial profiles in (28). The resultant eigenvalue $\omega=\omega_{r}+\mathrm{i} \gamma$ is that $\omega_{r}=-1.52$ and $\gamma=0.45$. This eigenvalue is the same as that in the calculation of $w_{0}(x)=1.0$ and $0 \leq x \leq 1$ instead of Eq. (28). The linear growth rate $\gamma$ is plotted in Fig. 3, where the agreement of linear theory with the simulation results is good. The linear analysis shows that $\omega_{r}=-3.04$ and $\gamma=0.43$ for $m=2$ mode.

Figure 5(a) plots the eigenfunction of $m=1$ mode of $\phi_{(m)}$ at $\tau=5.3$, and Fig. 5(b) plots the corresponding eigenfunction which is determined by the nonlocal eigenvalue equations, where the zeroth order quantities given in Eq. (28) are used. Because a classical diffusion of $D_{f}, T_{f}$, and $w_{f}$ is included in the simulation which is not included in the linear theory
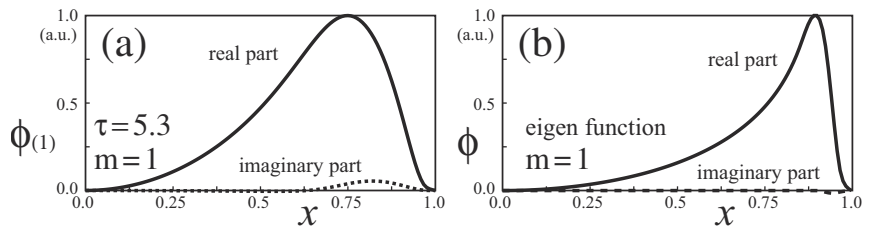

FIG. 5. The eigenfunction $\phi_{(m)}$ of $m=1$ mode. (a) is observed in the simulation at $\tau=5.3$ and (b) is calculated by the nonlocal linear analysis.

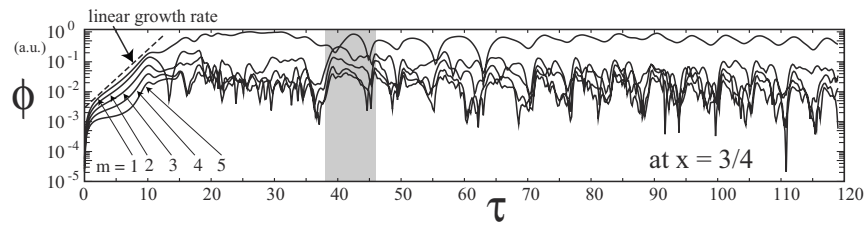

FIG. 6. The time evolution of $m=1-5$ modes of $\phi_{(m)}$ measured at $x=3 / 4$.

except for the second term in the right-hand side of Eq. (15), the perturbed electrostatic potential $\phi_{(m)}$ of $m=1$ has a mild radial profile compared with Fig. 5(b).

The long time behavior of $\phi_{(m)}$ is plotted in Fig. 6. The nonlinear phase follows after $\tau=10$. As is seen in Fig. 6, Fourier amplitudes of all $m$ modes behave in the same way.

(a)



(b)
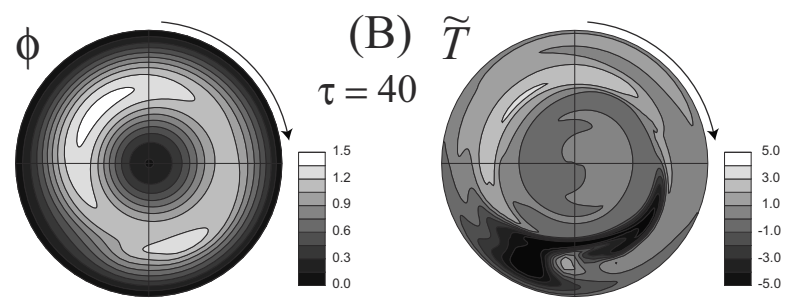

(c)
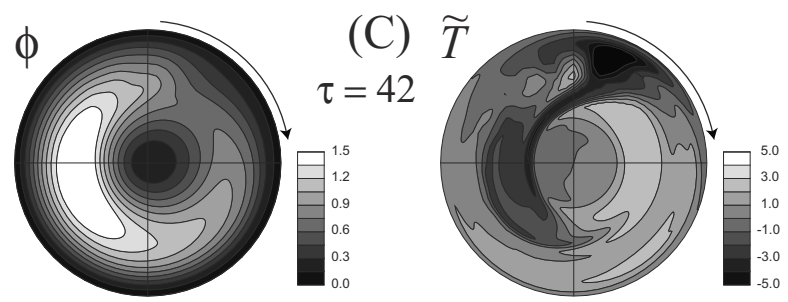

(d)

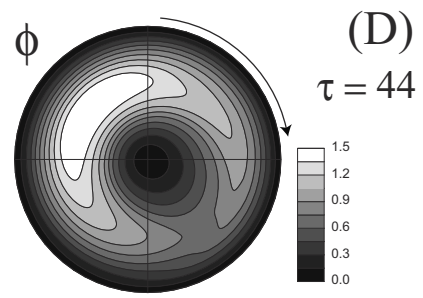

(D) $\widetilde{T}$

(e)
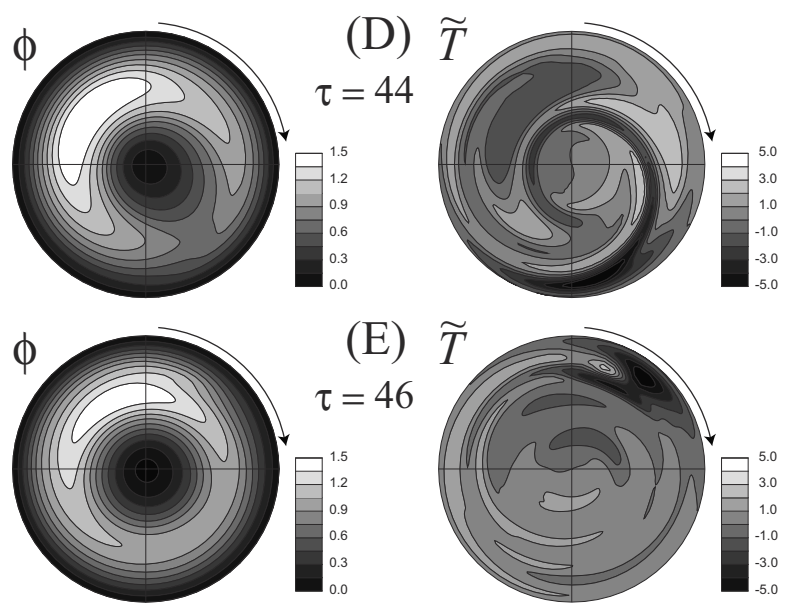

(E)

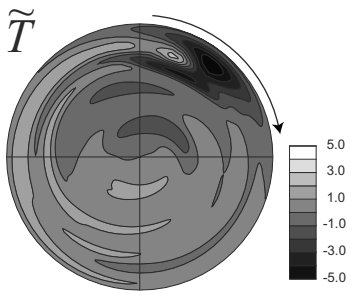

FIG. 7. The profiles of $\phi(x, \varphi)$ and $\tilde{T}(x, \varphi)=T_{f}(x, \varphi) / u(x)^{2 / 3}$ on a cross section of the magnetic flux tube at various time $\tau$. Here $\tilde{T}$ is a temperature on the cross section but not integrated over the magnetic flux tube. Note that $\tilde{T}(x, \varphi)$ excludes $T_{E}(x)$ from $T(x, \varphi)=T_{E}(x)+\epsilon^{2} T_{f}(x, \varphi)$, while $\phi(x, \varphi)$ includes the zeroth electrostatic potential $\phi_{(0)}(x)$ in it. 

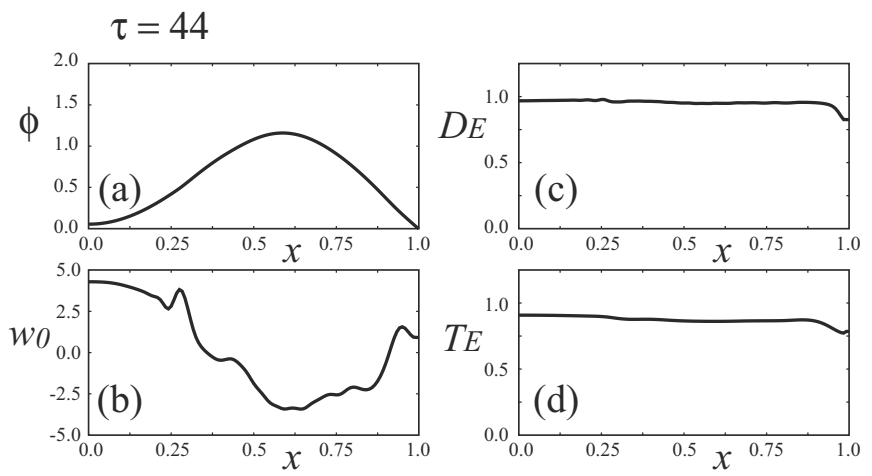

FIG. 8. The radial profiles at $\tau=44$ of (a): potential $\phi$, (b): specific dynamic vorticity $w_{0}$, (c): mass density $D_{E}$, and (d): effective temperature $T_{E}$.

That is, the patterns that all modes grow and decay are repeated periodically in Fig. 6 .

This pattern is seen more clearly in Fig. 7, which is the time evolution of electrostatic potential $\phi(x, \varphi)$ and equicontours of perturbed temperature $\widetilde{T}(x, \varphi)$ at $\tau=38-46$. This time zone corresponds to the shaded region of Fig. 6 . In the nonlinear phase the electrostatic potential has a local minimum point around the center which is contrast to the initial monotonous radial profile with a local maximum point at $x=0$ in Fig. 4(a). Plasma rotates clockwise in a period of $\tau \simeq 4$ at the periphery which is almost the same as $\omega_{r}$ of $m=1$ flute mode in a linear phase. On the other hand, the plasma rotates counterclockwise near the axis. The radial profiles of the zeroth components $\phi_{0}(x)$ obtained from Figs. 7(a)-7(e), which have one maximum at $x=0.6 \sim 0.7$, are stable and continue to the end of the simulation run which is shown in Fig. 8. Here, Fig. 8 plots the radial profiles of $\phi_{0}$, $w_{0}, D_{E}$, and $T_{E}$ observed in the simulation at $\tau=44$, and it is seen that the magnitudes of $D_{E}(x)$ and $T_{E}(x)$ decrease in time due to the radial loss resulting from the flute mode fluctuations as well as the classical diffusion.

The low temperate region at the periphery at $\tau=38$ in Fig. 7(A) makes an invasion upon the core region along an equipotential surface in time in Figs. 7(B)-7(D), and then go back to the original situation of Fig. $7(\mathrm{~A})$ at $\tau=46$ in Fig. $7(\mathrm{E})$. The time sequence of Figs. $7(\mathrm{~A})-7(\mathrm{E})$ is repeated in Fig. 6 and it causes the nondiffusive radial transport. Therefore, the diffusion due to the flute modes results from the plasma convection.

The simulation with the initial condition $w_{0}(x)=+1.0$ $(0 \leq x \leq 1)$ has exactly the same behavior in the

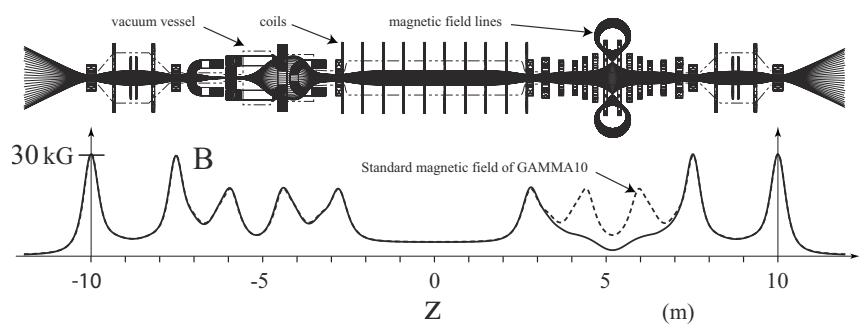

FIG. 9. The schematic diagram of the GAMMA10 tandem mirror with divertor, where one nonaxisymmetric minimum- B (anchor) mirror cell is replaced by an axisymmetric divertor mirror cell.

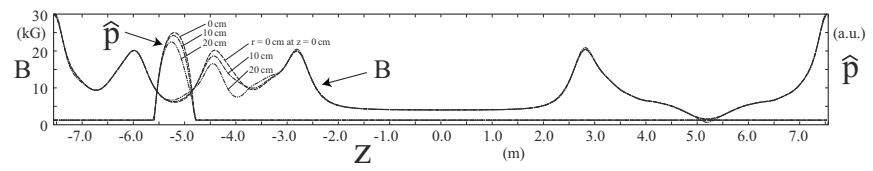

FIG. 10. The profiles of pressure $\hat{p}$ and magnetic field $B$ along a magnetic field line which passes through $r=0,10$, and $20 \mathrm{~cm}$ at $z=0 \mathrm{~cm}$, respectively. The separatrix magnetic field line passes at $(r, z) \simeq(21.6 \mathrm{~cm}, 0 \mathrm{~cm})$ and at $(r, z) \simeq(47.7 \mathrm{~cm}, 520.0 \mathrm{~cm})$ where the magnetic null point is.

linear phase as the simulation with the initial condition $w_{0}(x)=-1.0(0 \leq x \leq 1)$, except that the profiles of perturbed quantities such as $T_{f}(x, \varphi)$ are antisymmetric and the signs of $\phi$ and $w_{f}$ are opposite. The nonlinear steady state with the initial condition $w_{0}(x)=+1.0(0 \leq x \leq 1)$ agrees with that of $w_{0}(x)=-1.0(0 \leq x \leq 1)$.

\section{FLUTE-MODE FLUCTUATIONS IN THE GAMMA10 WITH DIVERTOR}

The GAMMA10 tandem mirror ${ }^{9}$ is working in a long time after it was built in 1981. So the plan to include an axisymmetric divertor magnetic field configuration in the GAMMA10 coil system is in progress. The first plan is to install the divertor coils in the central cell. ${ }^{19}$ However, in the recent design, one anchor minimum-B magnetic field is to be replaced by a divertor magnetic field, which is plotted in Fig. 9. The coil design has to solve the problems on the budget, equilibrium, stability, transport, etc. Figure 9 is a candidate at present although it is not a final design.

One important problem on a divertor mirror is to make clear the effects of flute mode fluctuations on the plasma transport in a nonlinear state. We use Eqs. (11)-(13), (15)(17), and (19), which were derived on an assumption of the axisymmetric magnetic field, in order to investigate the flute mode fluctuations in the nonlinear phase. In order to apply these equations to the effectively axisymmetrized GAMMA10 with divertor, we change the definition of the specific volume $U$ from Eq. (2) to Eq. (5) with the pressure axial profile

$$
\hat{p}(B) \equiv \hat{p}_{\perp}(B)+\hat{p}_{\|}(B)=\max \left[\frac{p_{A}}{D_{E}(0) T_{E}(0)} \frac{\left(B_{m}^{2}-B^{2}\right)}{\left(B_{m}^{2}-B_{c}^{2}\right)}, 1\right] .
$$

Here $B_{c}$ is the magnetic field at the anchor midplane on axis, and $B_{m}=1.7 B_{c}$, so that $\hat{p}(B)$ has a maximum value of $p_{A} /\left[D_{E}(0) T_{E}(0)\right]$ at $B=B_{c}$. The pressure profile (29) is applied to the anchor region $(-600 \mathrm{~cm}<z<-450 \mathrm{~cm})$, and the pressure $\hat{p}(B)=1$ is assumed in the other axial region, which is shown in Fig. 10. The pressures $\hat{p}_{\perp, \|}(B)$ are the separation of variables $\hat{p}_{\perp, \|}(B)=p_{\perp, \|}(\psi, B) / \nu(\psi)$ which are used in Eq. (4). The mass density $D_{E}(x)$ and temperature $T_{E}(x)$ are normalized to be unity at $x=0$ as an initial condition (at $\tau=0)$. Strictly speaking, the basic Eqs. (11)-(13) and (15)-(17) contain two different effective $u$. One is pressureweighted $u$ defined by Eqs. (5) and (29), the other is densityweighted $u$ which are the terms proportional to $D_{f} / u$ and $D_{E} / u$. However the strict distinction of these two effective $u$ complicates the problem, and that the axial profiles of pres- 


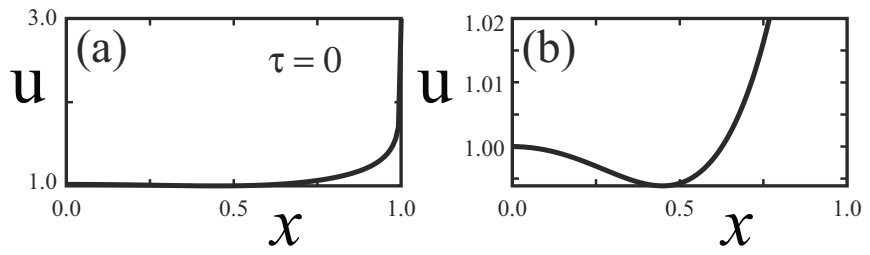

FIG. 11. The radial profiles of specific volume $u(x)$ in case of $p_{A}=20$. (a) plots the specific volume where the magnetic field line of radius $x=1$ passes at $(r, z) \simeq(47.5 \mathrm{~cm}, 520.0 \mathrm{~cm})$. (b) magnifies $u(x)$ in the region $x \lessgtr 3 / 4$ in (a).

sure and mass density remain unknown experimentally. Therefore the longitudinal profiles of pressure and mass density are assumed to be the same in this article and all $u$ appearing explicitly in the basic Eqs. (11)-(13) and (15)-(17) are replaced by the effective $u$ defined by Eqs. (5) and (29).

In the GAMMA10 tandem mirror the magnetic field $B$ is almost constant radially at the central midplane, but $B$ changes radially in the transition region between anchor mirror and central solenoid regions. Figure 10 plots the profiles of $\hat{p}(B)$ along a magnetic field line of different radii in the case of $p_{A}=20$ in Eq. (29), which are used to calculate the specific volume of a magnetic field line in Eq. (5). The resultant specific volume is plotted in Fig. 11. The specific volume is recalculated every interval of $\delta \tau=0.1$ with $D_{E}(0) T_{E}(0)$ obtained in the simulation at that time $\tau$. Note that the specific volume $u(x)$ is normalized to be unity at $x=0$ every time $\tau$ by Eq. (14). It is seen that there is a good curvature region in $0<x<0.4$ in Fig. 11 because $\partial u / \partial x<0$ is satisfied there.

The initial conditions adopted here are that $D_{E}(x)=1.0$, $T_{E}(x)=\exp \left\{-2 x^{2}\right\} u(x)^{\gamma-1}, w_{0}(x)=+1.0$, and the small initial perturbation given by Eq. (27) is added to $T_{f}(x)$. The boundary conditions are the same as those in Sec. IV. The external sources of $Q_{T 0}(x)$ and $Q_{D 0}(x)$ are taken into account in order to investigate the flute mode fluctuations in the steady state, that is, $Q_{T 0}(x)=\exp \left\{-2 x^{2}\right\}$ and $Q_{D 0}(x)=0.5 \exp \left\{-x^{2}\right\}$ in Eqs. (12) and (13). Other external sources are assumed to be 0 , i.e., $Q_{w 0}(x)=0$ and $\widetilde{Q}_{w}(x)=\widetilde{Q}_{D}(x)=\widetilde{Q}_{T}(x)=0$. Time step $\Delta t=5 \times 10^{-5}$, and the $(x \times \varphi)=\left(121 \times 2^{6}\right)$ meshes are used. The expansion parameter $\epsilon^{2}=10^{-2}$ is chosen.

Figure 12 plots the time evolution of $m=1-5$ Fourier amplitudes of $\phi_{(m)}(x)$ at $x=1 / 2$. The $m=1$ mode of $\phi_{(m)}$ grows in time at $\tau \simeq 125$. The linear growth rate $\gamma$ in Fig. 12 is evaluated by the nonlocal linear analysis described in Sec. III, the result of which is $\omega=\omega_{r}+\mathrm{i} \gamma$ with $\omega_{r}=0.28$ and $\gamma=0.12$, where the radial profiles $u, D_{E}, T_{E}$, and $w_{0}$ in

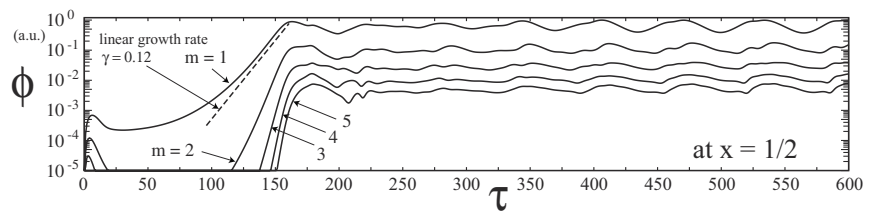

FIG. 12. The time evolution of $m=1-5$ modes of $\phi_{(m)}$ measured at $x=1 / 2$. The dashed lines in the figure are the linear growth rates, where $u$, $D_{E}, T_{E}$, and $w_{0}$ obtained in the simulation are used in the analysis.
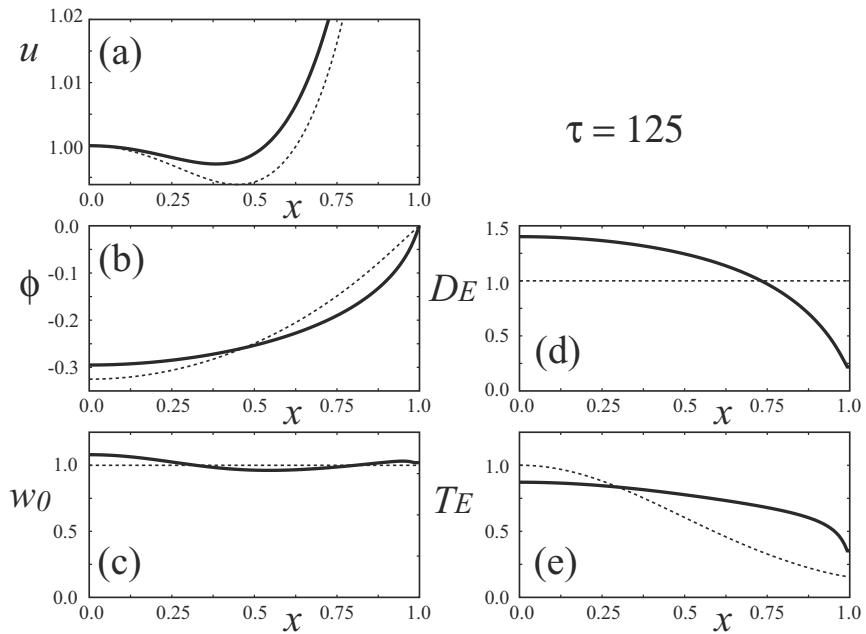

FIG. 13. The radial profiles at $\tau=125$ of (a) specific volume, (b) potential $\phi$, (c) specific dynamic vorticity $w_{0}$, (d) mass density $D_{E}$, and (e) effective temperature $T_{E}$. Here the dashed line in each figure is the initial profile.

Fig. 13 are used in the analysis. The simulation enters the nonlinear phase after $\tau=160$ and the steady state is realized.

Figure 13 plots the profiles (solid lines) obtained by the simulation at $\tau=125$ and the initial profiles (dashed lines). The mass density $D_{E}(x)$ profile shows that the mass density source $Q_{D 0}(x)$ makes $D_{E}(x)$ larger than unity near $x=0$ and radial loss across $x=1$ makes it lower near $x=1$. In the linear growing phase, the flute modes do not enhance the radial transport so much, and the classical diffusion makes the temperature $T_{E}(x)$ profile flat in the core region and makes it steep near $x=1$. The specific volume $u(x)$ is calculated every interval of $\delta \tau=0.1$ by Eq. (29) during the simulation run. Here $\hat{p}(B)$ in Eq. (29) is proportional to $p_{A} /\left[D_{E}(0) T_{E}(0)\right]$, where $D_{E}(0) T_{E}(0)$ is determined by the simulation at each time. So the time dependence of $u(x)$ results from the modification of longitudinal profile of $\hat{p}_{\|, \perp}(B)$. The magnetic well calculated by solid line (which is proportional to $-\partial u / \partial x$ ) becomes shallower than the initial well in Fig. 13(a) because the $D_{E}(0) T_{E}(0)$ is larger at $\tau=125$ than the initial magnitude.

The radial profile of $m=1$ Fourier amplitude of $\phi_{(m)}(x)$ at $\tau=125$ is plotted in Fig. 14(a). The eigenfunction evaluated by the nonlocal linear analysis by using the radial profiles $u, D_{E}, T_{E}$, and $w_{0}$ in Fig. 13 is plotted in Fig. 14(b). Although the eigenfunction is a little bit broader in the simulation than that in the linear analysis, both real part of eigenfunctions agrees with each other.

The effects of the flute mode fluctuations on the transport can be seen in Fig. 15. Here
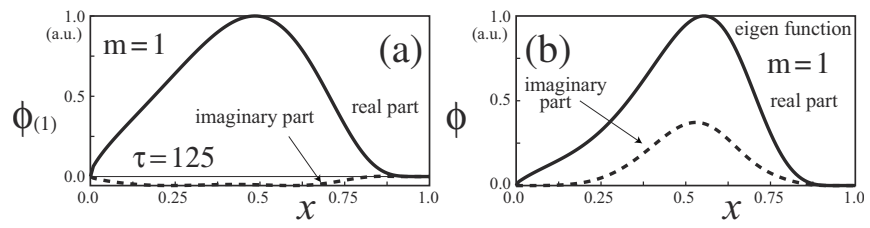

FIG. 14. The eigenfunction $\phi_{(m)}$ of $m=1$ mode. (a) is observed in the simulation at $\tau=3$ and (b) is calculated by the nonlocal linear analysis. 
$\langle\mathrm{DE}\rangle$

(a)

$\langle\mathrm{TE}\rangle$

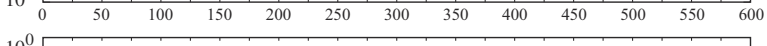

(b)

$\langle\mathrm{DET} \mathrm{T}\rangle$

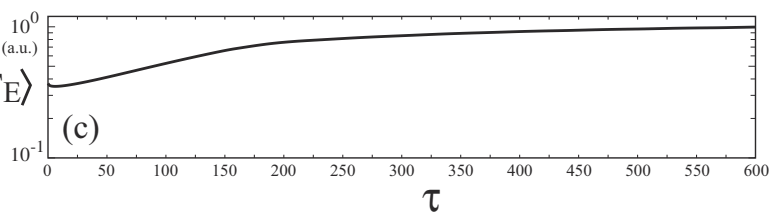

FIG. 15. The time evolution of total density, total temperature, and total energy (total entropy).

$$
\begin{aligned}
& \left\langle D_{E}\right\rangle \equiv \int_{0}^{1} D_{E}(x) x \mathrm{~d} x, \quad\left\langle T_{E}\right\rangle \equiv \int_{0}^{1} T_{E}(x) x \mathrm{~d} x, \\
& \left\langle D_{E} T_{E}\right\rangle \equiv \int_{0}^{1} D_{E}(x) T_{E}(x) x \mathrm{~d} x .
\end{aligned}
$$

Noting that $D_{E}(x)$ and $T_{E}(x)$ are the quantities integrated along a magnetic field line, the quantities $\left\langle D_{E}\right\rangle$ and $\left\langle D_{E} T_{E}\right\rangle$ represent the total mass number and total internal energy (total entropy). Note that $\left\langle D_{E}\right\rangle$ and $\left\langle T_{E}\right\rangle$ as well as $\left\langle D_{E} T_{E}\right\rangle$ change by only the fluxes across $x=1$ and source terms in Eqs. (12) and (13). The quantities $\left\langle D_{E}\right\rangle,\left\langle T_{E}\right\rangle$, and $\left\langle D_{E} T_{E}\right\rangle$ increase linearly in time before $\tau=180$ and then begin to saturate in the simulation. The saturation of the growth of these quantities begins when the flute instability enters the nonlinear phase in Fig. 12.

The influence of the flute mode fluctuations in the nonlinear phase on the radial transport of $T_{E}$ can be seen in Fig. 16, which plots the equicontour surfaces of the potential $\phi$ and temperature $T(x, \varphi) \equiv\left[T_{E}(x)+\epsilon^{2} T_{f}(x, \varphi)\right] / u(x)^{2 / 3}$ at $\tau=500 \sim 570$. Here $T(x, \varphi)$ is the temperature on the $(x, \varphi)$ plane, not integrated over the magnetic flux tube. Figure 16 is composed of pairs of $\phi(x, \varphi)$ and $T(x, \varphi)$, for example, Figs. 16(a) and 16(A) are a pair of $\phi$ and $T$ observed at $\tau=500$ in the simulation. As is seen in these figures the plasma rotates counterclockwise in the entire region (in the direction of arrow in the figure) in a period of $\tau \simeq 34$. The maximum of $T$ (white colored segment) in Fig. 16(A) increases in Fig. 16(C) and then enters the decreasing phase in Fig. 16(F), and enters again the increasing phase of $T$. These increment and decrement of $T$ is repeated in the nonlinear phase. The remarkable feature in Fig. 16 is that the equicontours of $\phi$ do not coincide with the equicontours of $T$, so the internal energy losses radially through $\boldsymbol{E} \times \boldsymbol{B}$ drifts. (a)

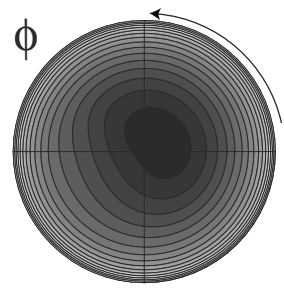

(b)

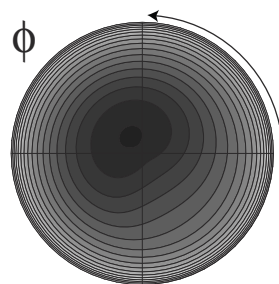

(c)

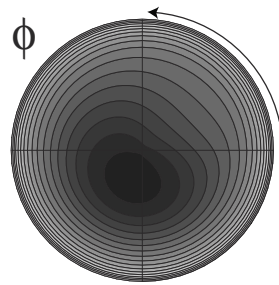

(d)
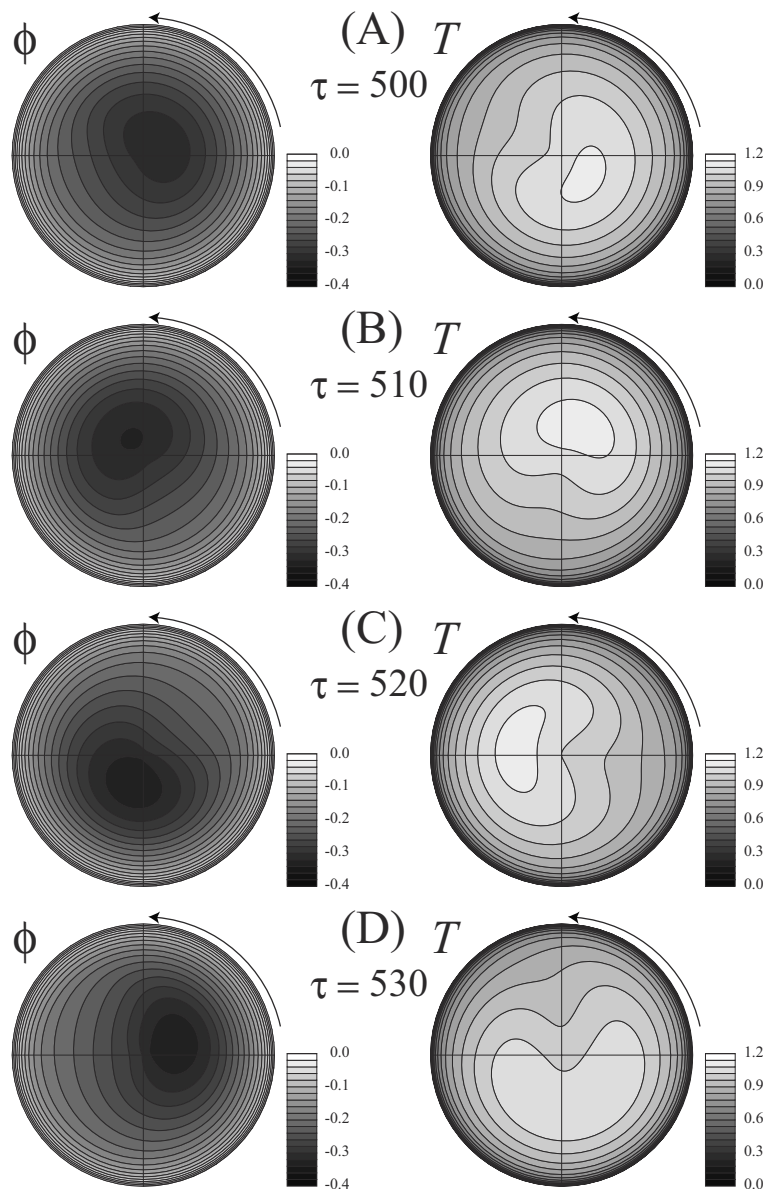

(B)

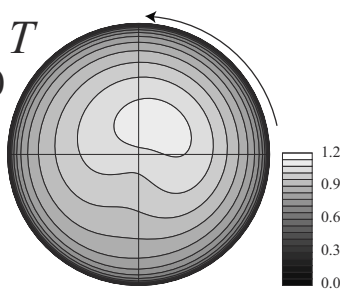

(C) $\tau=520$

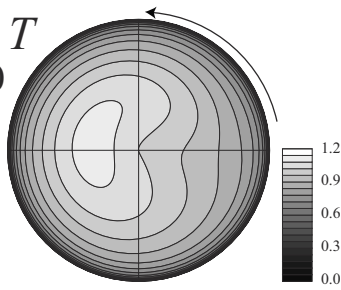

(h)

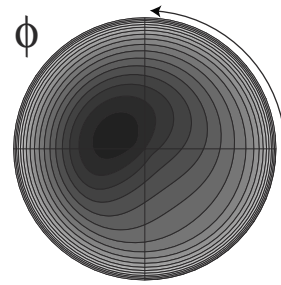

(f)

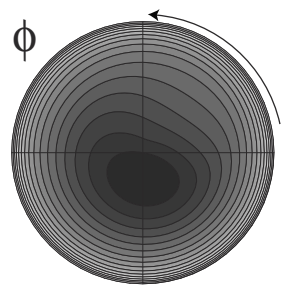

(g)

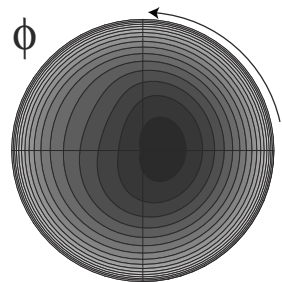

(G)
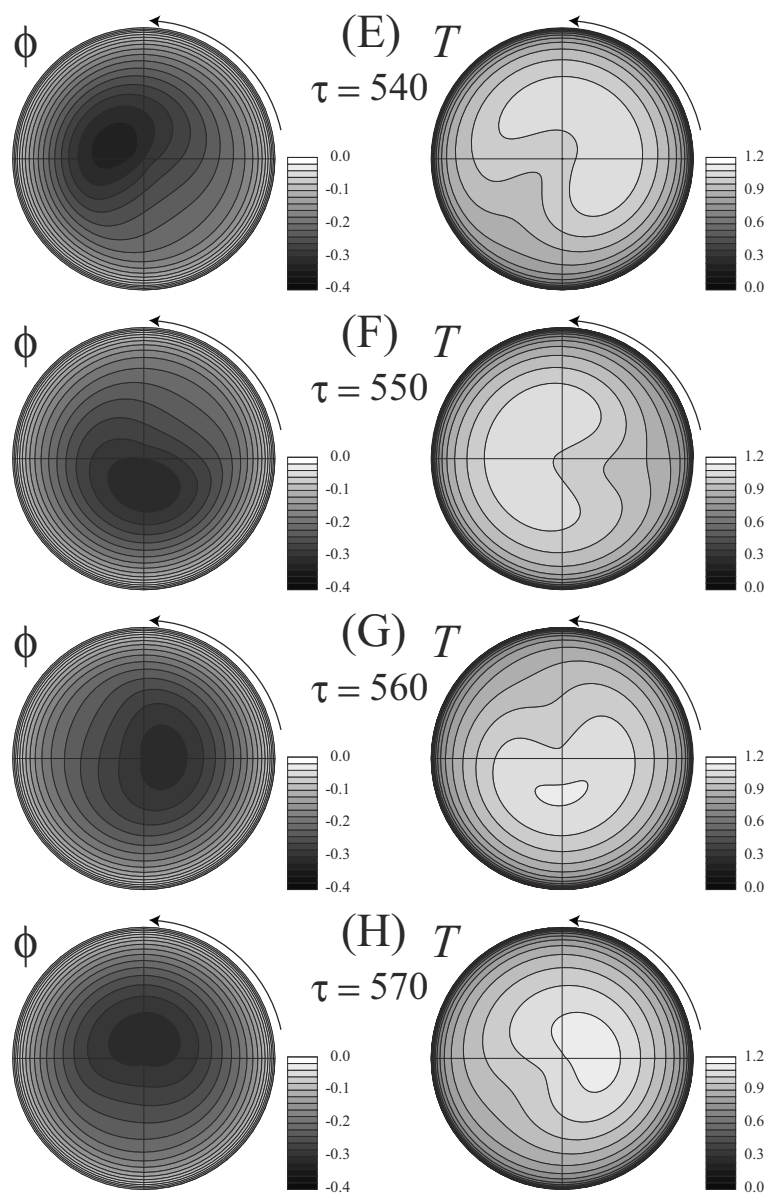

(H)

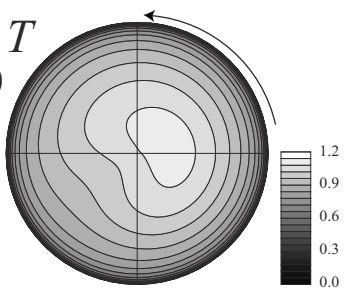

FIG. 16. The profiles of $\phi(x, \varphi)$ and $T(x, \varphi)=\left[T_{E}(x)+\epsilon^{2} T_{f}(x, \varphi)\right] / u(x)^{2 / 3}$ on a cross section of the magnetic flux tube at various time $\tau$. Here $T$ is a temperature on the cross section, but not integrated over the magnetic flux tube, and $\phi(x, \varphi)$ includes the zeroth electrostatic potential $\phi_{0}(x)$ in it. 

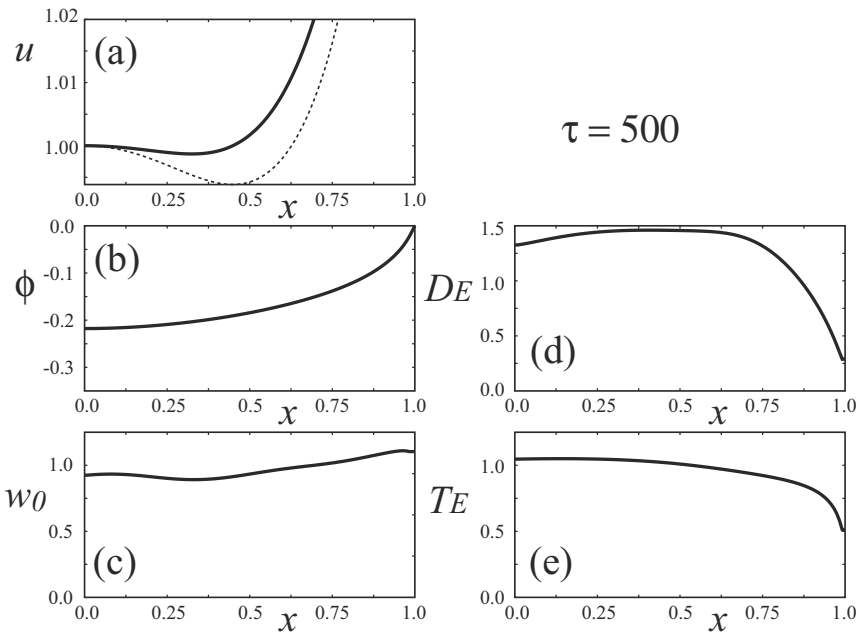

FIG. 17. The radial profiles at $\tau=500$ of (a) specific volume, (b) potential $\phi$, (c) specific dynamic vorticity $w_{0}$, (d) mass density $D_{E}$, and (e) effective temperature $T_{E}$. The dashed line in (a) is the initial profile of $u(x)$.

Figure 17 is the radial profiles of various quantities in the nonlinear steady state at $\tau=500$. In this phase the specific volume $u$ in Fig. 17(a) has a profile without any magnetic well, and $D_{E}(x) T_{E}(x)$ is almost constant radially in the core region.

\section{SUMMARY AND DISCUSSION}

We have developed the computer simulation code to investigate the flutelike mode fluctuations in the open magnetic divertor with the basic equations in Sec. II. This code can evaluate the fluctuation associated with the presence of nonuniform plasma flows (similar to the Kelvin-Helmholtz instability).

This code was applied to the model divertor in Sec. IV and the GAMMA10 with divertor in Sec. V. The growth rate of $m=1$ flute mode observed in the simulation agrees well with the nonlocal linear analysis in Sec. III and the eigenfunction of the mode in the simulation agrees with the linear analysis in both divertor cases.

It is first found in this article that there is a nonlinear steady state of GAMMA10 with divertor and this state is very different from the nonlinear steady state of model divertor. While the nonlinear state of GAMMA10 with divertor balances the sources $D_{D 0}, Q_{T 0}$, and the radial losses, in the nonlinear state of mode divertor $D_{E}$ and $T_{E}$ decay in time due to without any sources.

One different point of the nonlinear steady state is that the potential with one peak point at $x \simeq 0.6$ is formed in the model divertor in Fig. 8(a), while the potential with one peak at $x=0$ is realized in the GAMMA10 with divertor in Fig. 17(a). The local maximum segment (white colored segment) of $\phi$ existed at $x=0$ initially in Fig. 4(a) was pushed out to the off-axis by the flute instability and then the profile of $\phi$ in Fig. 7(a) was formed.

Another different point is that a large flute instability occurs repeatedly at almost a fixed interval in the nonlinear steady state in Fig. 6, while the remarkable flute instability is not observed in Fig. 12, although both cases cause a large radial transport. The reason why those different nonlinear steady states are realized in the different divertor cases is a future problem to be solved.

As mentioned in Sec. I the stability can only be achieved for the case of gradually decreasing pressure radial profile in a divertor cell, ${ }^{22,35}$ which is essentially different from the average minimum-B mirror stabilization. In the simulation of Sec. V on GAMMA10 with divertor, however, a steep $T_{E}(x)$ radial profile shown by dotted line in Fig. 13(e) is chosen as an initial condition, and sources $Q_{D 0}(x)$ and $Q_{T 0}(x)$ are also steep. Although these initial condition and sources with never gradually decreasing radial profiles are chosen, the very stable nonlinear steady state is realized. That is, the flute mode fluctuations have an effect of making the initial pressure radial profile a stable profile.

\section{ACKNOWLEDGMENTS}

The authors wish to acknowledge the valuable discussions with Dr. V. P. Pastukhov.

${ }^{1}$ L. S. Hall and B. McNamara, Phys. Fluids 18, 552 (1975).

${ }^{2}$ G. I. Dimov, V. V. Zakaidakov, and M. E. Kishinevskij, Sov. J. Plasma Phys. 2, 326 (1976).

${ }^{3}$ T. K. Fowler and B. G. Logan, Comments Plasma Phys. Controlled Fusion 2, 167 (1977).

${ }^{4}$ D. D. Ryutov and G. V. Stupakov, Fiz. Plazmy 4, 501 (1978); Sov. J. Plasma Phys. 4, 278 (1978).

${ }^{5}$ D. D. Ryutov and G. V. Stupakov, Dokl. Akad. Nauk SSSR 240, 1086 (1978); Sov. Phys. Dokl. 23, 412 (1978).

${ }^{6}$ R. H. Cohen, Nucl. Fusion 19, 1579 (1979).

${ }^{7}$ I. Katanuma, Y. Kiwamoto, S. Adachi, M. Inutake, K. Ishii, K. Yatsu, K. Sawada, and S. Miyoshi, Nucl. Fusion 27, 2041 (1987).

${ }^{8}$ I. Katanuma, Y. Kiwamoto, K. Ishii, K. Yatsu, and S. Miyoshi, Phys. Fluids B 1, 1459 (1989).

${ }^{9}$ M. Inutake, T. Cho, M. Ichimura, K. Ishii, A. Itakura, I. Katanuma, Y. Kiwamoto, Y. Kusama, A. Mase, S. Miyoshi, Y. Nakashima, T. Saito, A. Sakasai, K. Sawada, I. Wakaida, N. Yamaguchi, and K. Yatsu, Phys. Rev. Lett. 55, 939 (1985).

${ }^{10}$ For example, K. Miyamoto, Plasma Physics for Nuclear Fusion (MIT, Cambridge, Mass., 1979).

${ }^{11}$ M. N. Rosenbluth and C. L. Longmire, Ann. Phys. 1, 120 (1957).

${ }^{12}$ T. B. Kaiser and L. D. Pearlstein, Phys. Fluids 26, 3053 (1983).

${ }^{13}$ T. B. Kaiser, W. M. Nevins, and L. D. Pearlstein, Phys. Fluids 26, 351 (1983).

${ }^{14}$ I. Katanuma, Y. Tatematsu, K. Ishii, T. Tamano, and K. Yatsu, J. Phys. Soc. Jpn. 69, 3244 (2000).

${ }^{15}$ D. D. Ryutov, Proceedings of the Societá Italiana di Fisica, Course and Workshop, Villa Monastero, Varenna, Italy, 1987 (unpublished) Vol. II, pp. 791-815.

${ }^{16}$ A. V. Burdakov, A. A. Ivanov, and E. P. Kruglyakov, Trans. Fusion Technol. 51, 17 (2007).

${ }^{17}$ E. P. Kruglyakov, G. I. Dimov, A. A. Ivanov, and V. S. Koidan, Trans. Fusion Technol. 47, 1 (2005).

${ }^{18}$ R. F. Post, T. K. Fowler, R. Bulmer, J. Byers, D. Hua, and L. Tung, Trans. Fusion Technol. 47, 49 (2005).

${ }^{19}$ I. Katanuma, Y. Sasagawa, Y. Tatematsu, Y. Nakashima, T. Cho, and V. P. Pastukhov, Nucl. Fusion 46, 608 (2006).

${ }^{20}$ V. V. Arsenin and A. Yu. Kuyanov, Plasma Phys. Rep. 26, 741 (2000).

${ }^{21}$ V. V. Arsenin and A. Yu. Kuyanov, Trans. Fusion Technol. 39, 175 (2001).

${ }^{22}$ V. V. Arsenin and A. Yu. Kuyanov, Plasma Phys. Rep. 27, 635 (2001).

${ }^{23}$ B. Lane, R. S. Post, and J. Kesner, Nucl. Fusion 27, 277 (1987).

${ }^{24}$ V. P. Pastukhov and A. Yu. Sokolov, Fiz. Plasmy 17, 1043 (1991); Sov. J. Plasma Phys. 17, 603 (1991).

${ }^{25}$ Y. Sasagawa, I. Katanuma, Y. Mizoguchi, T. Cho, and V. P. Pastukhov, Phys. Plasmas 13, 122506 (2006).

${ }^{26}$ V. P. Pastukhov, Trans. Fusion Technol. 47, 138 (2005).

${ }^{27}$ J. A. Casey, B. G. Lane, J. H. Irby, K. L. Brau, S. N. Golovato, W. C. 
Guss, J. Kesner, P. S. Post, E. Sevillano, and J. Zielinski, Phys. Fluids 31, 2009 (1988).

${ }^{28}$ Y. Yasaka, M. Takano, and H. Takeno, Trans. Fusion Technol. 39, 350 (2001).

${ }^{29}$ A. C. England, D. K. Lee, S. G. Lee, M. Kwon, S. W. Yoon, Y. Yasaka, N. Sugimoto, I. Katanuma, K. Yashiro, and T. Imai, Nucl. Fusion 49, 125008 (2009).

${ }^{30}$ V. P. Pastukhov, Fiz. Plazmy 31, 628 (2005); Plasma Phys. Rep. 31, 577 (2005).

${ }^{31}$ I. Katanuma, V. P. Pastukhov, T. Imai, M. Ichimura, T. Kariya, Y. Nakashima, H. Hojo, R. Minami, Y. Yamaguchi, M. Yoshikawa, H. Akao,
T. Watanabe, and W. Horton, J. Plasma Fusion Res. 84, 279 (2008).

${ }^{32}$ S. I. Braginskii, in Reviews of Plasma Physics, edited by A. M. A. Leontovich (Consultants Bureau, New York, 1965), Vol. 1.

${ }^{33}$ V. P. Pastukhov, Trans. Fusion Technol. 51, 34 (2007).

${ }^{34}$ For example, D. Potter, Computational Physics (Wiley-Interscience, London, 1973).

${ }^{35}$ V. P. Pastukhov, K. Ishii, Y. Tatematsu, T. Saito, Md. K. Islam, Y. Nakashima, and T. Cho, Trans. Fusion Technol. 47, 276 (2005); Proceedings of the International Conference on Open Magnetic Systems for Plasma Confinement, Novosibirsk, Russia, 5-9 July 2004 (unpublished). 